\title{
Validation of Age Estimation Methods Using Pulpal Volume Changes in Radiographs for Korean Adults
}

\author{
Tae-Hoon Lee, Jung-Hun Hong, Sang-Seob Lee, Jeong-Seung Kwon, Jong-Hoon Choi \\ Department of Orofacial Pain and Oral Medicine, College of Dentistry, Yonsei University, Seoul, Korea
}

Received March 15, 2014

Revised March 28, 2014

Accepted April 10, 2014
Purpose: It aims to verify the applicability of existing age estimation methods derived from data of foreign population groups to Korean population groups. Moreover it is to suggest a new way applicable to practical age estimation on the basis of newly calculated regression formulae from data of Korean population groups and develop a subsidiarily applicable method to the existing method.

Methods: Ratio of pulp cavity to dental crown was calculated by measuring the height and width of dental crowns and pulp cavities at the cervical line from 4,034 first and second upper molars, first and second upper premolars, first and second lower molars and first and second lower premolars on both left and right sides of 400 patients who had been treated in Dental Hospital of Yonsei University College of Dentistry, and regression equations were derived from the values of the ratio.

Results: The equation with correlation coefficients the highest among females was as follows: age $=107.96-75.684 \times \# 17$ TCHI $-53.741 \times \# 26$ TCVI-40.664×\#45 TCVI-56.307 $\times 46$ TCVI. Randomized anohter Korean female subjects $(n=20)$ are applicated to the new equation. Mean of error of estimate is 10.322 years, standard deviation is 12.852 years. Minimum of error of estimate is 1.018 years, maximum is 21.365 years

Conclusions: The error range of age estimation was found to be slightly wider when the existing regression formulae of Drusini were applied to Korean population groups. Also age estimation in females using the ratio of pulp cavity to dental crown measured with the length and width of dental crowns and pulp cavities from maxillomandibular molars was observed to have the highest reliability in the research. However, we consider that advanced equations of regression are needed to apply to both molars and premolars of males and females in the future.

Key Words: Age estimation; Korean population group; Panoramic radiography; Tooth-coronal index

\section{INTRODUCTION}

The main purpose of forensic odontology is to determine unidentified human bodies by comparing and analyzing their dental features recorded before and after their death. Teeth are recognized as highly identifiable body organs since they have an excellent physical and chemical resistance and they last longer than any other tissues in a decomposed condition. In addition, each of 32 teeth has their own anatomical and morphological characteristics and shows random features obtained from dental treatments through their life time as well. Applications of age estimation are not only limited to forensic identification. It is also applied as a scientific evidence to verify ages of immigrants due to increasing international immigration and mediate conflicts generated from social phenomena such as school enrollment, welfare benefaction, employment and marriage. ${ }^{1)}$

Approaches of forensic age estimation should be differentiated according to an age group of human subjects. They

Copyright (c) 2014 Korean Academy of Orofacial Pain and Oral Medicine. All rights reserved. 
are classified into childhood, adolescence and adulthood. In childhood, mixed dentition appears divided to odontogenesis (development) and odontoptosis (falling) of deciduous teeth and odontogenesis (development) and succession of permanent teeth. Development of teeth is recognized as a useful index for evaluation of physical maturity. ${ }^{2,3)}$ Various methods to determine age have been derived from evaluating degrees of teeth development, analyzing its maturity and comparing the results to chronological age due to the low variability of individuals in teeth development. Since Nolla ${ }^{4)}$ introduced a method for age determination based on the degree of teeth development in 1960 for the first time, Demirjian et al. ${ }^{5)}$ suggested criteria for evaluation of teeth development securing a higher objectivity and presented a method of age estimation quantifying levels of teeth development into scores in 1973. Most of age estimation for children has been derived based on data of each population group with the criteria of teeth development by Demirjian et al. ${ }^{5}$ because of its rationality and usability.

Age determination for adulthood after mid twenties is examined by evaluating and analyzing the degree of regressive changes with age appeared in teeth. In 1950, Gustafson ${ }^{6}$ suggested the six histological features of teeth presenting regressive changes: the degree of attrition, periodontal involution, secondary dentin formation, cementum deposition, transparent dentine formation and root resorption, and he derived regression formulae from them. In most of the published methods of age estimation, they do not adopt all of the six histological features of Gustafson, ${ }^{6}$ they mostly use one of the features to estimate age. At present, the most-used method in Korea is the one measuring and analyzing the degree of transparent dentin formation ${ }^{7)}$ and the other mixing measurements of periodontal involution and transparent dentine. ${ }^{8)}$

Since Bodecker ${ }^{9)}$ wrote an article of the correlation between secondary dentine and chronological age in 1925, there have been many academic achievements of its research. According to the research of Johanson ${ }^{10)}$ in 1971, he confirmed a high coefficient of the correlation between secondary dentine and chronological age with 0.63 which is the second highest value after 0.84 of the correlation coefficient between transparent dentin and chronological age. The formation of secondary dentine continues throughout life starting right after dentine formation is complete. Most of secondary dentine is deposited at the pulpal side of primary dentine, and the size of the pulp cavity decreases with age. ${ }^{6,10-12)}$ Secondary dentine is divided to regular and irregular dentine (or tertiary dentine); regular secondary dentine is deposited continuously with aging while irregular secondary dentine (or tertiary dentine) is laid down in pathological conditions. Secondary dentine is distinguished from primary dentine, for it has only few dentinal tubules. But, when dentinal tubules are found in secondary dentine occasionally, they are arranged more irregularly in irregular secondary dentine. ${ }^{13)}$ There is a research result that the degree of the formation of regular secondary dentine is consistent in the same dentition regardless of the degree of attrition. ${ }^{14)}$ Other researcher said that it is more affected by aging rather than attrition or external stimulus. ${ }^{13)}$

In age estimation of adulthood, methods applicable to living individuals are limited since invasive methods which need dental extraction cannot be applied. Therefore, for now, it is limited to use the degree of attrition inevitably in the department of oral medicine at university hospitals. The formation of secondary dentine has received far less research attention and it has been difficult to apply to practical use while various researches with the degree of attrition have been actively conducted in Korea. Although it is difficult to observe and evaluate the formation of secondary dentine in living individuals, it can be identified with the degree of pulp cavity reduction on radiographs at second hand. Kvaal et al. ${ }^{15)}$ developed an age estimation method, analyzing the reduction of pulp cavity from various angles with measurements between diverse measuring points and ratio conversed from the measurements after radiographing the root apex of central incisors, lateral incisors and second premolars of maxilla, and lateral incisors, canines and first premolars of mandible. The author proved a high correlation between age and the reduction of pulp cavity in the research. It presented a significant result to evaluate the degree of pulp cavity due to the increase of secondary dentine; however, it cannot be applied to multi rooted teeth since it researched on single rooted teeth only.

In 1985, Ikeda et al. ${ }^{16)}$ developed a new index, called the tooth-coronal index (TCI), with radiography. They defined cervical line as a straight line connecting between 
cementoenamel junctions and divided anatomical dental crown and root at the line. They set clear criteria of measurement for multi rooted teeth such as molars with the coronal height $(\mathrm{CH})$, defined as the vertical distance from the cervical line to the highest cusp, and the coronal pulp cavity height ( $\mathrm{CPCH})$, designated as the distance between the cervical line and the highest pulp horn. TCI was calculated from dividing $\mathrm{CPCH}$ by $\mathrm{CH}$ and then multiplying that value by 100 , and they observed the correlation between age and the index. In 1997, Drusini et al. ${ }^{17)}$ found the correlation between age and TCI and derived equations for age estimation by measuring the lower premolars and molars from radiographs of 433 individuals and calculating TCI. In the research, they found a high correlation between TCI and age ranged from 0.77 to 0.85 and confirmed its possibility of high applicability for practical use with \pm 6 years of the standard error.

Researchers in Korea have published literatures about the correlation between the size of pulp chamber and age in the past. In 1981, Chung and $\mathrm{Kim}^{18)}$ took note of the age estimation method using the area ratio between pulp cavity and external feature of tooth. They radiographed 1,208 teeth of upper and lower central incisors, lateral incisors, canine, first premolars and second premolars and calculated their areas in two dimensions after making their sextuple size prints. They classified ages from 21 to 70 by 5 years and reported that it is practically applicable to upper and lower anterior teeth. In 1993, Hong and Ko ${ }^{19)}$ classified 838 individuals of males and females from early twenties to early sixties into nine groups by age and took intraoral radiographs of them with paralleling method. They divided the radiographs into groups by 4 levels: level A (the normal level), level B (the mild calcification level), level C (the moderate calcification level), and level D (the severe calcification level), graded them as 1, 2, 3, or 4. However, most of them discussed the correlation between the morphological change of pulp chamber and age only and it was rather difficult to apply to practical age estimation. Therefore, we measured 4,034 teeth from radiographs of more than 400 Korean adults in this research and verified if the foreign methods for age estimation are applicable to Korean population groups, using TCI of Ikeda et al. ${ }^{16)}$ and the area measurement of pulp cavity at the cervical of Kvaal et al. ${ }^{15)}$ We chose significant teeth and measurement methods from measurements of the two methods and determined the applicability of index, calculated from the changes of pulp cavity with age in a new combination of the previous methods for age estimation of Korean.

\section{MATERIALS AND METHODS}

\section{Materials}

We measured and analyzed a sample consisting of panoramic X-ray photographs of 4,034 teeth including upper right first and second molars and premolars, upper left first and second molars and premolars, lower right first and second molars and premolars and lower left first and second molars and premolars from 400 individuals who have been treated at Dental Hospital of Yonsei University College of Dentistry from September to November in 2013, made up of each 40 males and females randomly extracted from age groups of 20s, 30s, 40s, 50s, and 60s.

We started the research with permission from the Bioethics Council of Yonsei University since it used radiographs originated from human bodies (IRB approval No. 2-2012-0032). We paid attention to prevent any unnecessary disclosure of private information except age and sex in a process of the research.

The research excluded the following teeth presented on panoramic radiographs: carious teeth, fractured teeth, restored teeth due to class II caries, restored teeth due to class I caries but the depth of its restorative material is deep and teeth without a clear sight of its criteria due to the overlap of its major site with other teeth or bones.

\section{Methods}

We measured the width and height of dental crown and pulp cavity at the cervical line and calculated the ratio of pulp cavity to dental crown. The tooth-coronal vertical index (TCVI), calculated from the ratio of dental crown height to pulp cavity height, and the tooth-coronal horizontal index (TCHI), derived from the width ratio of dental crown to pulp cavity, were used as variables to estimate age (Fig. 1).

The panoramic radiographs were measured and analyzed with $\pi$-View Star (Infinitt Healthcare Co., Ltd., Seoul, Korea). For precise measurement, they were observed with their 


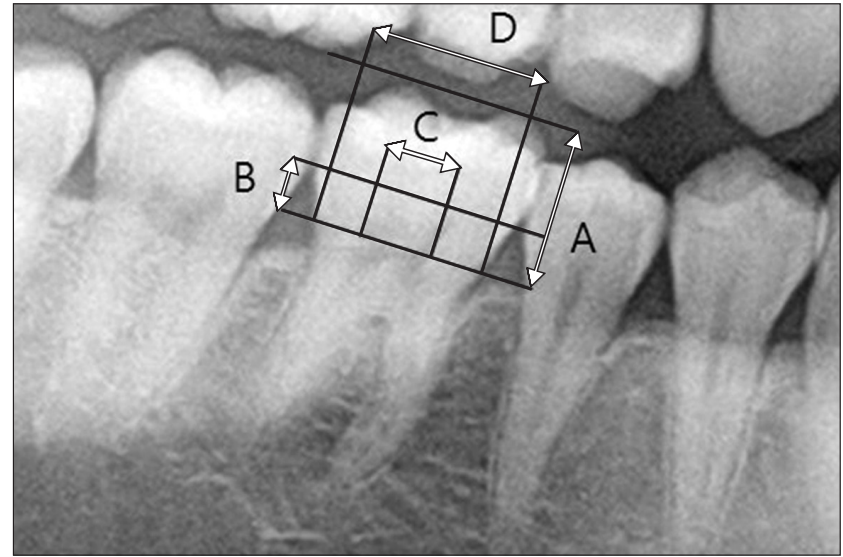

Fig. 1. Methods of teeth measurement on radiographs. We measured the distance from the mesiodistalcementoenamel junction to the highest cusp ('A') and to the highest pulp horn ('B') as well as the width of pulp cavity and dental crown at the cervical line. The values of 'B/A' (tooth-coronal vertical index) and 'C/D' (toothcoronal horizontal index) were taken as a variable for age estimation.

sextuple-size enlargement and measured by limb length and caliper which are subordinate menus of the internal measurement tool in the program. According to the calculation of TCI by Drusini et al., ${ }^{17)}$ the cusp height of pulp horn and teeth was measured at the highest point setting the cervical line (the cementoenamel junction) as a base line for limb length. In addition, the width of dental crown and pulp cavity at the cervical line was measured with caliper along the cervical line which was the base line for limb length.

The measurement ratio of pulp cavity to dental crown was used, because panoramic radiographs always distort the images of objects to be enlarged or scaled down in some degrees, and, therefore, the measurement of height and width on radiographs cannot be applied as it is. We analyzed the ratio using group correspondence analysis, variance analysis, linear regression analysis and step analysis with PASW Statistics 18.0 program (IBM Co., Armonk, NY, USA).

\section{RESULTS}

The following results were obtained from 4,034 teeth of 400 patients in 20-69 years (mean \pm standard deviation, $44.37 \pm 14.46$ years).

Paired t-test was conducted to confirm the difference between measurements of teeth on both sides from the same
Table 1. Comparison of both side index

\begin{tabular}{ccc}
\hline Index & Mean \pm SD & p-value \\
\hline TCVI & & \\
$\# 14-\# 24$ & $0.008 \pm 0.109$ & 0.409 \\
$\# 15-\# 25$ & $0.020 \pm 0.100$ & 0.029 \\
$\# 16-\# 26$ & $0.002 \pm 0.079$ & 0.757 \\
$\# 17-\# 27$ & $0.019 \pm 0.082$ & 0.009 \\
$\# 34-\# 44$ & $0.011 \pm 0.093$ & 0.202 \\
$\# 35-\# 45$ & $0.013 \pm 0.091$ & 0.103 \\
$\# 36-\# 46$ & $0.003 \pm 0.076$ & 0.690 \\
$\# 37-\# 47$ & $0.006 \pm 0.079$ & 0.389 \\
TCHI & & \\
$\# 14-\# 24$ & $0.003 \pm 0.077$ & 0.661 \\
$\# 15-\# 25$ & $0.009 \pm 0.064$ & 0.117 \\
$\# 16-\# 26$ & $0.007 \pm 0.079$ & 0.366 \\
$\# 17-\# 27$ & $0.012 \pm 0.074$ & 0.082 \\
$\# 34-\# 44$ & $0.020 \pm 0.052$ & 0.000 \\
$\# 35-\# 45$ & $0.015 \pm 0.056$ & 0.004 \\
$\# 36-\# 46$ & $0.007 \pm 0.051$ & 0.118 \\
$\# 37-\# 47$ & $0.003 \pm 0.069$ & 0.666 \\
\hline
\end{tabular}

$\mathrm{TCVI}$, tooth-coronal vertical index; $\mathrm{TCHI}$, tooth-coronal horizontal index; SD, standard deviation.

person; the test confirmed statistical difference $(\mathrm{p}<0.05)$ between the left and right side with TCVI of second upper premolars and molars and TCHI of first and second lower premolars as shown on Table 1. In consequence of the result, regression analysis was conducted using the left and right teeth as different significant indexes.

To verify the applicability of the regression formulae of Drusini (the regression formulae for premolars: age= 77.617-1.4636xTCVI, the regression formulae for molars: age $=76.073-1.4576 \times \mathrm{TCVI}$ ) to actual age estimation of Korean, we analyzed the deviation between calculated age and chronological age substituting TCVI of Korean. As a result, it was considered not to be applicable to Korean in the forensic age estimation with the deviation average from minimum 11.578 years (\#26) to maximum 17.013 years (\#44) and the standard deviation from minimum 8.615 years to maximum 11.908 years which is considered slightly large (Fig. 2).

The correlation between age and each dental index (TCHI, TCVI) was found as shown on the Table 2, 3. To derive more significant regression formulae for age estimation, step analysis was conducted in combination with the indexes higher than 0.2 which represents a relatively high correlation. We examined the difference among the indexes from each age group of 20s, 30s, 40s, 50s, and 60s to confirm 


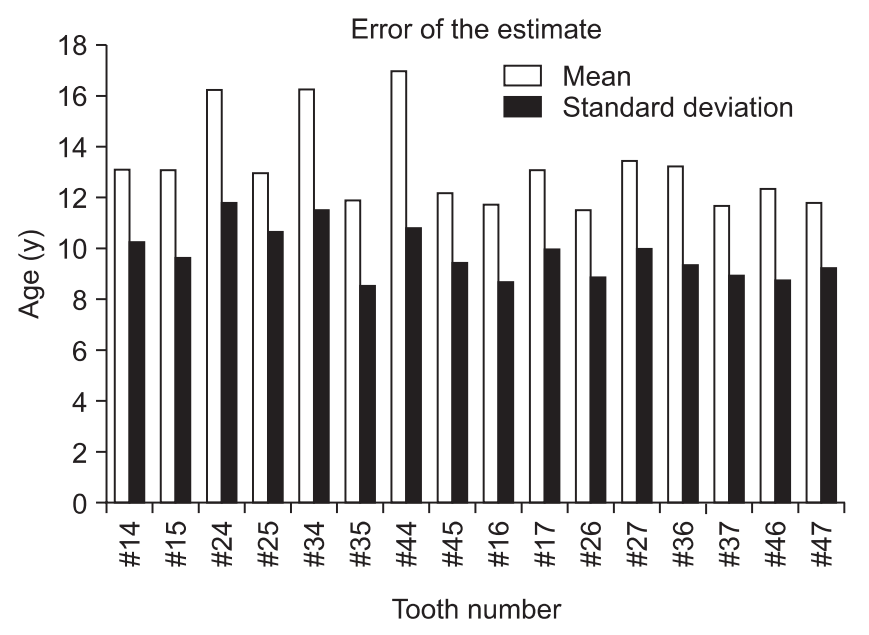

Fig. 2. Application of Drusini formulae to the Korean population.

the difference of significance among the indexes by age, but none of significant difference was found among age groups.

Step analysis is a method making regression equations with indexes which are statistically significant to age only, and the results of the regression formulae with indexes of the correlation coefficients higher than 0.2 are presented on the Table 4 . The equation with correlation coefficients the highest among females was as follows: age $=107.96-$ 75.684x\#17 TCHI-53.741×\#26 TCVI-40.664×\#45 TCVI56.307×\#46 TCVI.

Randomized another Korean female subjects $(n=20)$ are applicated to the new equation. Mean of error of estimate is 10.322 years, standard deviation is 12.852 years. Minimum of error of estimate is 1.018 years, maximum is 21.365 years.

\section{DISCUSSION}

For deposition of secondary dentine, many of previous researchers have presented morphological changes of pulp cavity anatomically and reductions of dental crown and pulp cavity in size with radiological and histological findings of adult teeth. The research of Kvaal et al. ${ }^{15}$ is one of the most recognized foreign researches regarding deposition of secondary dentine. They measured the size of dental crown and pulp from intraoral radiographs of incisors, canines and premolars and found high coefficients of determination between the size and age ranged from 0.56 to 0.76 . Although the research derived significant results to evaluate
Table 2. Correlation coefficient between TCVI and age

\begin{tabular}{|c|c|}
\hline Tooth number & Correlation coefficient \\
\hline \multicolumn{2}{|l|}{ Male+female } \\
\hline$\# 14$ & 0.0893 \\
\hline$\# 15$ & 0.0727 \\
\hline$\# 16$ & 0.1580 \\
\hline$\# 17$ & 0.0555 \\
\hline$\# 24$ & 0.0010 \\
\hline$\# 25$ & 0.0453 \\
\hline$\# 26$ & 0.1567 \\
\hline$\# 27$ & 0.0137 \\
\hline \#34 & 0.0407 \\
\hline \#35 & 0.2166 \\
\hline \#36 & 0.1716 \\
\hline$\# 37$ & 0.1508 \\
\hline$\# 44$ & 0.0160 \\
\hline$\# 45$ & 0.1716 \\
\hline$\# 46$ & 0.2209 \\
\hline$\# 47$ & 0.1354 \\
\hline \multicolumn{2}{|l|}{ Male } \\
\hline$\# 14$ & 0.0559 \\
\hline$\# 15$ & 0.0229 \\
\hline$\# 16$ & 0.0598 \\
\hline$\# 17$ & 0.0190 \\
\hline$\# 24$ & 0.0004 \\
\hline$\# 25$ & 0.0023 \\
\hline$\# 26$ & 0.0506 \\
\hline$\# 27$ & 0.0076 \\
\hline \#34 & 0.0016 \\
\hline \#35 & 0.1371 \\
\hline$\# 36$ & 0.1140 \\
\hline \#37 & 0.1035 \\
\hline \#44 & 0.0007 \\
\hline$\# 45$ & 0.1005 \\
\hline \#46 & 0.1630 \\
\hline$\# 47$ & 0.0808 \\
\hline \multicolumn{2}{|l|}{ Female } \\
\hline$\# 14$ & 0.1300 \\
\hline \#15 & 0.1666 \\
\hline$\# 16$ & 0.2833 \\
\hline$\# 17$ & 0.1082 \\
\hline$\# 24$ & 0.0020 \\
\hline$\# 25$ & 0.1740 \\
\hline$\# 26$ & 0.3241 \\
\hline$\# 27$ & 0.1193 \\
\hline$\# 34$ & 0.1332 \\
\hline$\# 35$ & 0.3118 \\
\hline$\# 36$ & 0.2320 \\
\hline$\# 37$ & 0.2103 \\
\hline \#44 & 0.0595 \\
\hline$\# 45$ & 0.2831 \\
\hline$\# 46$ & 0.2850 \\
\hline \#47 & 0.1977 \\
\hline
\end{tabular}

TCVI, tooth-coronal vertical index. 
Table 3. Correlation coefficient between TCHI and age

\begin{tabular}{|c|c|}
\hline Tooth number & Correlation coefficient \\
\hline \multicolumn{2}{|l|}{ Male+female } \\
\hline$\# 14$ & 0.2188 \\
\hline$\# 15$ & 0.2136 \\
\hline \#16 & 0.1211 \\
\hline$\# 17$ & 0.2514 \\
\hline \#24 & 0.1677 \\
\hline$\# 25$ & 0.2368 \\
\hline$\# 26$ & 0.1560 \\
\hline$\# 27$ & 0.1989 \\
\hline \#34 & 0.2521 \\
\hline$\# 35$ & 0.2802 \\
\hline \#36 & 0.1735 \\
\hline \#37 & 0.1551 \\
\hline \#44 & 0.1305 \\
\hline \#45 & 0.1703 \\
\hline \#46 & 0.1156 \\
\hline$\# 47$ & 0.1412 \\
\hline \multicolumn{2}{|l|}{ Male } \\
\hline$\# 14$ & 0.2371 \\
\hline$\# 15$ & 0.1241 \\
\hline$\# 16$ & 0.0875 \\
\hline$\# 17$ & 0.1368 \\
\hline$\# 24$ & 0.0974 \\
\hline$\# 25$ & 0.1660 \\
\hline \#26 & 0.1312 \\
\hline$\# 27$ & 0.1557 \\
\hline$\# 34$ & 0.1825 \\
\hline \#35 & 0.2538 \\
\hline$\# 36$ & 0.1725 \\
\hline$\# 37$ & 0.1210 \\
\hline \#44 & 0.0642 \\
\hline$\# 45$ & 0.1778 \\
\hline \#46 & 0.0938 \\
\hline \#47 & 0.1456 \\
\hline \multicolumn{2}{|l|}{ Female } \\
\hline$\# 14$ & 0.2257 \\
\hline$\# 15$ & 0.3153 \\
\hline$\# 16$ & 0.1497 \\
\hline$\# 17$ & 0.3607 \\
\hline$\# 24$ & 0.2697 \\
\hline$\# 25$ & 0.3356 \\
\hline$\# 26$ & 0.1925 \\
\hline$\# 27$ & 0.2651 \\
\hline$\# 34$ & 0.3280 \\
\hline$\# 35$ & 0.3161 \\
\hline$\# 36$ & 0.1687 \\
\hline$\# 37$ & 0.1931 \\
\hline$\# 44$ & 0.2094 \\
\hline$\# 45$ & 0.1704 \\
\hline \#46 & 0.1439 \\
\hline$\# 47$ & 0.1501 \\
\hline
\end{tabular}

TCHI, tooth-coronal horizontal index.
Table 4. Step analysis of selective formation with tooth number, $\mathrm{TCHI}$ and TCVI

\begin{tabular}{lcc}
\hline \multicolumn{1}{c}{ Index } & $\mathrm{R}^{2}$ & $\begin{array}{c}\text { SE of the } \\
\text { estimate }\end{array}$ \\
\hline Male+female & & \\
\#17 TCHI, \#34 TCHI & 0.247 & 13.571 \\
\#17 TCHI, \#34 TCHI, \#46 TCVI & 0.291 & 13.166 \\
\#17 TCHI, \#34 TCHI, \#46 TCVI, \#27 TCHI & 0.321 & 12.883 \\
\#17 TCHI, \#46 TCVI & 0.265 & 13.555 \\
\#17 TCHI, \#46 TCVI, \#27 TCHI & 0.306 & 13.172 \\
\#17 TCHI, \#25 TCHI & 0.272 & 13.634 \\
\#17 TCHI, \#25 TCHI, \#27 TCHI & 0.291 & 13.455 \\
\#35 TCHI, \#35 TCVI & 0.265 & 13.491 \\
\#35 TCHI, \#35 TCVI, \#34 TCHI & 0.298 & 13.181 \\
\#34 TCHI, \#46 TCVI & 0.299 & 13.520 \\
\#34 TCHI, \#46 TCVI, \#35 TCVI & 0.323 & 13.291 \\
\#34 TCHI, \#46 TCVI, \#27 TCHI & 0.311 & 12.981 \\
\#46 TCVI, \#27 TCHI & 0.304 & 12.968 \\
Female & & \\
\#17 TCHI, \#46 TCVI & 0.582 & 10.588 \\
\#17 TCHI, \#15 TCHI & 0.538 & 10.805 \\
\#17 TCHI, \#36 TCVI, \#15 TCHI & 0.582 & 10.268 \\
\#17 TCHI, \#46 TCVI, \#26 TCVI & 0.653 & 9.651 \\
\#17 TCHI, \#46 TCVI, \#26 TCVI, \#45 TCVI & 0.684 & 9.205 \\
\#45 TCVI, \#35 TCVI & 0.527 & 10.895 \\
\#45 TCVI, \#35 TCVI, \#24 TCHI & 0.544 & 10.501 \\
\#17 TCHI, \#26 TCVI & 0.534 & 10.821 \\
\#17 TCHI, \#26 TCVI, \#15 TCHI & 0.597 & 10.067 \\
Male & & \\
\#27 TCHI, \#46 TCVI & 0.188 & 14.150 \\
\#35 TCHI, \#34 TCHI & 0.275 & 12.912 \\
\hline
\end{tabular}

$\mathrm{TCHI}$, tooth-coronal horizontal index; TCVI, tooth-coronal vertical index; SE, standard error.

the degree of pulp cavity reduction in size due to the increase of secondary dentine, it is still limited to apply to multi rooted teeth since its subjects were single rooted teeth only.

On the other hand, Drusini et al. ${ }^{17)}$ took note of the method of Ikeda et al. ${ }^{16)}$ and confirmed the correlation between TCI and age by radiographing 68 premolars and 98 molars of adults, instead of taking histological samples. The correlation coefficients were ranged from -0.73 (female molars) to -0.89 (female premolars), and the standard error was ranged from 8.79 to 10.08 years.

However, some researchers have insisted that the degree of secondary dentine deposition can be affected by not only age but also surrounding environment or genetic factor. Bang ${ }^{20)}$ said that weather can affect the deposition of secondary dentine, and Woods et al. ${ }^{21)}$ presented that it can be affected by diverse variables such as dietary habit. In 
addition, Kvaal et al. ${ }^{15)}$ suggested examining the accuracy of their work by asking various researchers to apply the regression formulae, derived by Kvaal et al., ${ }^{15)}$ to age groups of each population. Kanchan-Talreja et al. ${ }^{22)}$ examined the method of Kvaal et al. ${ }^{15)}$ using radiographs of root apex of Indian with the paralleling method and bisecting angle technique, but they suggested their own regression formulae due to the low applicability of Kvaal et al.'s method. ${ }^{15}$ Cameriere et al. ${ }^{23)}$ also verified the applicability of the regression formulae of Kvaal et al. ${ }^{15)}$ for Portuguese and derived a new equation. This research verified the applicability of the regression formulae of Ikeda and Drusini, using the size ratio between teeth and pulp from radiographs, to Korean population groups by applying measurements of Korean to the equations and comparing the outcomes with chronological age. Moreover we analyzed 4,034 teeth of 400 Korean to derive a new method of age estimation for Korean combining advantages of the Drusini and Kvaal's methods.

Although diverse radiography can be used to measure the structure of teeth, we planned the study with panoramic radiography to make it applicable for practical use often in spite of its disadvantage with a high possibility of image overlap of multiple bone structures and image distortion, because it is the most widely used radiography and it has a high usability taken frequently in general dental treatments. Radiologic methods to observe the change of pulp cavity in size due to the deposition of secondary dentine with age are periapical radiography, panoramic radiography, computed tomography (CT), etc.

Periapical radiography allows the most clear and precise analysis of a small number of teeth with ease. The shape and size of teeth on periapical radiographs can be different from those of the original teeth due to the distance between the teeth and X-ray tube, the angle of the tube when radiographed, the morphological change of the film as well as the morphology and location of the teeth. However, TCVI and TCHI, measured in this research, are not significantly affected by the distortion, since they calculate the width and height of dental crown and pulp cavity with ratio. Panoramic radiography is a familiar method for clinicians. It allows to record every maxillomandibular tooth on a film in a short period of time and to observe the total teeth at a look. It is also easy to observe pretreatment lesions and medical records of teeth on panoramic radiographs. But it has a lower resolution than periapical radiography especially for anterior teeth and it is often difficult to measure specific sites because it cannot be taken at the desired angle. Although CT allows diverse cross sections to be examined and makes it possible to research in three dimensions in comparison with the limits on directions of panoramic and periapical radiography, it is difficult to apply to clinicians and patients in terms of its equipment and cost. However age estimation method with $\mathrm{CT}$ has a high possibility to be used generally in the future, since age estimation is not encountered often in dental treatment and it has become popularized with the improved performance and lower cost due to the technological advance. Therefore we expect that researches regarding this method will be conducted from various angles in the future.

Many of equipment have become digitalized rapidly due to technological advance. One of the features is that images can be revised and enlarged with ease. The usability of measurement methods has been improved with measurement programs and it has become possible to measure more precisely with easily enlarged images. Moreover, in this research, we used the internal measurement method of the program, used for observation of panoramic radiography, to reduce image distortions and measurement errors among different programs. Especially, changes of measured values were prevented, and images kept their consistency using the internal ruler of the program when we controlled the level of enlargement, reduction, or contrast of images to find certain measuring points on radiographs.

Lower canines have been chosen for subjects of age estimation researches with the deposition of secondary dentine since most of them have only one relatively bigger root canal which allows more precise measurement and they normally last the longest. It is also because they are easy to analyze with less attrition, unlike molars attrited by food or anterior teeth worn by particular jobs, and they have the largest pulpal surface among single rooted teeth. On the other hand, multi rooted teeth and multi rooted canal are difficult to be read on radiographs by overlap, distortion and blur of the images. First and second premolars were particularly difficult to measure due to the axial angle of 
panoramic radiography causing slanted images. In this research, premolars were overlapped with their adjacent teeth and it was difficult to find the cementoenamel junctions of slanted teeth actually. Although it is believed that there is no difference between measurements of the left and right teeth, we found statistical significance mostly from premolars when we verified the difference between measurements of teeth on both sides of the same person. Premolars did not show difference of measurements by age and it was possibly caused by measurement errors due to the distortion and deformation of the images on radiographs. Therefore, Drusini et al. ${ }^{17)}$ took only 2-3 premolars and molars for measurement whose images were clear on each panoramic radiograph to avoid the errors.

In other words, significance was found partially as a result of analyzing the difference between TCVI and TCHI of both sides with his method and it became grounds of our suggestion to use each left and right tooth as variables of regression formulae, when age estimation was conducted with the equations derived from this research.

In addition, estimated age of the subjects of this research which was calculated with the measurement from their radiographs by Drusini's method was found to be slightly different from their chronological age and it appeared to be difficult to apply his method for practical use. It proved that there is a difficulty to directly apply foreign methods of age estimation to Korean, although they are meaningful methods for age estimation of their own population groups.

Moreover, both TCVI and TCHI, indexes of individual tooth, decreased with age in every age and sex, but their correlation coefficients were not statistically significant. Meanwhile, TCHI of every tooth presented higher or similar correlation coefficients compared with TCVI and it is possibly caused by the fact that TCHI measurements can be more precise than TCVI, since TCHI is affected more by the degree of attrition on the occlusal surface in case of molars. However calculating regression formulae with TCVI for premolars and TCHI for molars will be a meaningful research since TCVI is more usable for single rooted teeth for setting their measuring points.

Furthermore, the equation (age $=107.96-75.684 \times \# 17$ TCHI-53.741x\#26 TCVI-40.664x\#45 TCVI-56.307x\#46 TCVI), calculated in combination with components from
TCVI of Drusini and Ikeda and TCHI of Kvaal, was found to be relatively more significant in every age group of females. It is possible to apply the method to estimate ages of subjects which cannot be applied by other methods, such as burnt corpses, teeth with unrecognizable degrees of attrition, or radiographs only available for the estimation) and to reassess the estimation results after calculating with other regression formulae.

Finally, the result of this research proved that it is difficult to examine the degree of pulp cavity change in size by age on two-dimensional radiographs whose images are overlapped inevitably such as periapical radiographs, panoramic radiographs. Three-dimensional measurements by CT, etc can reduce the disadvantages of the two-dimensional radiography and there should be more studies regarding three-dimensional measurements in the future.

The aim of this research was to verify if the regression formulae for age estimation, derived from foreign population groups, can be applied to Korean population groups with 400 patients in ages of 20-69 years (200 males and 200 females) and to draw a new equation of regression which can present correlation between the size of pulp cavity and age. A sample totaling 4,034 intact teeth of the subjects (\#17, 272; \#27, 233; \#37, 276; \#47, 220; \#16, 273; \#26, 227; \#36, 277; \#46, 225; \#15, 275; \#25, 230; \#35, 286; \#45, 226; \#14, 271; \#24, 229; \#34, 288; \#44, 226) was chosen to measure $\mathrm{CH}$ in $\mathrm{mm}$ and $\mathrm{CPCH}$ in $\mathrm{mm}$, and the TCVI of Ikeda et al. ${ }^{16)}$ was calculated. The width of dental roots and pulps at the mesiodistal cementoenamel junction was measured, and the ratio of the measurements, named as the TCHI, was calculated and statistically analyzed. As a result, we derived conclusions as follows by applying the index to the age estimation method of the previous researcher (Drusini) using radiography.

1. The age estimation method of Drusini was not appropriate to apply to Korean population group (the average deviation, 11.578-17.013 years; the standard deviation, 8.61511.908 years).

2. In case of premolars and molars, the correlation between TCVI and age was found to be low (the maximum of 0.22).

3. In case of premolars and molars, the correlation between TCHI and age was found to be low (the maximum of 0.28).

4. The regression equation, age $=107.96-75.684 \times \# 17$ 
TCHI - 53.741×\#26 TCVI-40.664×\#45 TCVI-56.307×\#46

TCVI, was derived with the highest significance, using TCHI of upper right second molars, TCHI of upper right second premolars, and TCVI of lower right first molars with high correlation coefficients (higher than 0.6), among equations calculated in combination of TCVI and TCHI of Drusini and Kvaal.

In conclusions, it was not appropriate to apply the regression formulae derived from foreign population groups to Korean and the age estimation method, using pulp cavity of premolars and molars of Korean on panoramic radiographs, was considered to have a limited applicability for practical use.

\section{CONFLICT OF INTEREST}

No potential conflict of interest relevant to this article was reported.

\section{REFERENCES}

1. Willems G. A review of the most commonly used dental age estimation techniques. J Forensic Odontostomatol 2001;19:9-17.

2. Garn SM, Lewis AB, blizzard RM. Endocrine factors in dental development. J Dent Res 1965;44(Suppl):243-258.

3. Garn SM, Lewis AB, Kerewsky RS. Genetic, nutritional, and maturational correlates of dental development. J Dent Res 1965; 44(Suppl):228-242.

4. Nolla CM. The development of permanent teeth. J Dent Child 1960;27:254-266.

5. Demirjian A, Goldstein H, Tanner JM. A new system of dental age assessment. Hum Biol 1973;45:211-227.

6. Gustafson G. Age determination on teeth. J Am Dent Assoc 1950; 41:45-54.

7. Bang G, Ramm E. Determination of age in humans from root dentin transparency. Acta Odontol Scand 1970;28:3-35.

8. Lamendin H, Baccino E, Humbert JF, Tavernier JC, Nossintchouk $\mathrm{RM}$, Zerilli A. A simple technique for age estimation in adult corpses: the two criteria dental method. J Forensic Sci 1992;37: 1373-1379.

9. Bodecker CF. A consideration of some of the changes in teeth from young to old age. Dent Cosm 1925;67:543-549.

10. Johanson G. Age determination from human teeth. Odontol Revy 1971;22(Suppl 21):40-126.

11. Morse DR. Age-related changes of the dental pulp complex and their relationship to systemic aging. Oral Surg Oral Med Oral Pathol 1991;72:721-745.

12. Solheim T. Amount of secondary dentin as an indicator of age. Scand J Dent Res 1992;100:193-199.

13. Berkovitz BKB, Holland GR, Moxham BJ. A colour atlas and textbook of oral anatomy, histology and embryology. 2nd ed. London: Wolfe Publishing Ltd.; 1992.

14. Burns KR, Maples WR. Estimation of age from individual adult teeth. J Forensic Sci 1976;21:343-356.

15. Kvaal SI, Kolltveit KM, Thomsen IO, Solheim T. Age estimation of adults from dental radiographs. Forensic Sci Int 1995;74:175185.

16. Ikeda N, Umetsu K, Kashimura S, Suzuki T, Oumi M. Estimation of age from teeth with their soft X-ray findings. Nihon Hoigaku Zasshi 1985;39:244-250.

17. Drusini AG, Toso 0, Ranzato C. The coronal pulp cavity index: a biomarker for age determination in human adults. Am J Phys Anthropol 1997;103:353-363.

18. Chung ET, Kim CY. An estimation of age based on the changes in the human dental cavity caused by increase in age (by surface index of pulp cavity). Korean J Oral Med 1981;6:101-110.

19. Hong HC, Ko MY. A study of the change of pulp cavity of the adult posterior teeth in ageing. Korean J Oral Med 1993;18:107117.

20. Bang G. Age changes in teeth: developmental and regressive. In: Iscan MY, ed. Age markers in the human skeleton. Springfield: Charles C. Thomas Publisher Ltd.; 1989. pp. 211-235.

21. Woods MA, Robinson QC, Harris EF. Age-progressive changes in pulp widths and root lengths during adulthood: a study of American blacks and whites. Gerodontology 1990;9:41-50.

22. Kanchan-Talreja P, Acharya AB, Naikmasur VG. An assessment of the versatility of Kvaal's method of adult dental age estimation in Indians. Arch Oral Biol 2012;57:277-284.

23. Cameriere R, Cunha E, Sassaroli E, Nuzzolese E, Ferrante L. Age estimation by pulp/tooth area ratio in canines: study of a Portuguese sample to test Cameriere's method. Forensic Sci Int 2009;193:128.e1-e6. 